\title{
Decreased Expression of CD69 on T Cells in Tuberculosis Infection Resisters
}

\author{
Zhen-Yan Chen ${ }^{1+}$, Lei Wang ${ }^{21}$, Ling Gu', Rong Qu ${ }^{3}$, Douglas B. Lowrie ${ }^{1,4}$, Zhidong Hu ${ }^{1,4 *}$, \\ Wei Sha ${ }^{2 *}$ and Xiao-Yong Fan ${ }^{3,4 *}$ \\ 'Shanghai Public Health Clinical Center, Key Laboratory of Medical Molecular Virology of MOE/MOH, Fudan University, \\ Shanghai, China, ${ }^{2}$ Shanghai Pulmonary Hospital, Tongji University, Shanghai, China, ${ }^{3}$ School of Laboratory Medicine \\ and Life Science, Wenzhou Medical University, Wenzhou, China, ${ }^{4}$ TB Center, Shanghai Emerging and Re-emerging Institute, \\ Shanghai, China
}

OPEN ACCESS

Edited by:

Rogelio Hernandez Pando,

Instituto Nacional de Ciencias

Médicas y Nutrición Salvador Zubirán

(INCMNSZ), Mexico

Reviewed by:

Yvonne Rosenstein

National Autonomous University

of Mexico, Mexico

Jiezuan Yang

Zhejiang University, China

*Correspondence:

Zhidong Hu

huzhidong2008@163.com

Wei Sha

shfksw@126.com

Xiao-Yong Fan

xyfan008@fudan.edu.cn

${ }^{t}$ These authors have contributed

equally to this work

Specialty section:

This article was submitted to

Microbial Immunology,

a section of the journal

Frontiers in Microbiology

Received: 30 April 2020

Accepted: 20 July 2020

Published: 07 August 2020

Citation:

Chen Z-Y, Wang L, Gu L, Qu R, Lowrie DB, Hu Z, Sha W and Fan $X-Y$

(2020) Decreased Expression of CD69 on T Cells in Tuberculosis

Infection Resisters.

Front. Microbiol. 11:1901. doi: 10.3389/fmicb.2020.01901
Background: CD69 is a biomarker of T-cell activation status, but its activation status in human Mycobacterium tuberculosis (Mtb) infection remains elusive.

Methods: A set of cohorts of patients with different tuberculosis (TB) infection status including active TB patients (ATB), latent tuberculous infection patients (LTBI) and close contacts (CCs) of ATB was designed, and the expression profiles of CD69 and several T-cell markers were determined on Mtb antigen-stimulated T cells by flow cytometry.

Results: The frequencies of $\mathrm{CD}^{+}{ }^{+}$and $\mathrm{CD}^{+}{ }^{+} \mathrm{T}$ cells were both comparable among Mtb-infected individuals including ATB and LTBI, which guaranteed the consistency of the background level. A t-Distributed Stochastic Neighbor Embedding (tSNE) analysis on a panel of six phenotypic markers showed a unique color map axis gated on $T$ cells in the CCs group compared with ATB and LTBI populations. By further gating on cells positive for each individual marker and then overlaying those events on top of the tSNE plots, their distribution suggested that some markers were expressed differently in the CCs group. Further analysis showed that the expression levels of CD69 on both CD4 ${ }^{+}$ and $\mathrm{CD}^{+} \mathrm{T}$ cells were significantly lower in the CCs group, especially in interferon$\gamma$-responding $T$ cells.

Conclusion: Our findings suggest that the T-cell activation status of CD69 is associated with Mtb infection and may have the potential to distinguish LTBI from those populations who have been exposed continuously to Mtb but have not become infected.

Keywords: tuberculosis, CD69, T-cell, latent infection, close contacts, resister

\section{INTRODUCTION}

Tuberculosis (TB) is the leading cause of death due to a single infectious disease in the world, there were an estimated 10 million new TB cases and 1.5 million deaths in the year of 2018 (WHO, 2019). Host-directed therapy is a promising strategy for TB treatment but it has not yielded persuasive results due to our incomplete understanding of immunological mechanism against this disease.

About a quarter of the world's population has latent TB infection (LTBI), and their lifetime risk of developing TB disease is around 5\% 15\% (WHO, 2019). Mycobacterium tuberculosis (Mtb) 
infection is defined by standard clinical tests including tuberculin skin tests (TST) and interferon (IFN)- $\gamma$ release assays (IGRAs). Recently, it was reported that nine months of isoniazid or four months of rifampin treatment can prevent the development of active TB disease in persons with LTBI (Samanovic and Darwin, 2016), illustrating the feasibility of sterile eradiation of $M t b$ in latent infection. However, the exact risk and timing of disease resulting from the exposure of close contacts (CCs) to active TB patients (ATB) have not been determined (Reichler et al., 2018). Recently, particular attention has been placed on the group of CCs with persistently negative TST/IGRA results despite prolonged exposure, a group termed "resisters" (Simmons et al., 2018; Kaipilyawar and Salgame, 2019). Identification of differential epidemiological and immunological characteristics of the CCs that are either "resisters" or LTBI will provide greater biological insights to prevent or clear early $\mathrm{TB}$ infections and develop novel diagnostic methods.

$\mathrm{T}$ cells, especially Th1 CD4 ${ }^{+} \mathrm{T}$ cells, are a crucial part of anti-TB immunity. T-cell activation and exhaustion represent the different immune status at different stages of $M t b$ infection, distinguished by the expression of specific biomarkers. Our previous work demonstrated that the expression of KLRG1, regarded as a T-cell terminal differentiation biomarker, was associated with the progression of human $\mathrm{TB}$ ( $\mathrm{Hu}$ et al., 2018). In animal infection/vaccination models, the expression of several T-cell biomarkers, such as CD38 (Dintwe et al., 2013), CD69 (Andersen and Smedegaard, 2000; Kauffman et al., 2018), CTLA-4 (Kirman et al., 1999; Nandakumar et al., 2014), LAG-3 (Workman et al., 2004; Phillips et al., 2015), Tim-3 (Jayaraman et al., 2016), and PD-1 (Lazar-Molnar et al., 2010; Reiley et al., 2010), has been reported to be related to TB infection. However, association with clinical TB has not been shown for all of them, especially their expression profiles in CCs remain to be defined.

In this study, the expression profiles of six biomarkers related to TB infection were compared among ATB, LTBI and CCs populations by flow cytometry in a designed cohort. As a result, Although the frequency of $\mathrm{T}$ cells was identical among these groups, a t-Distributed Stochastic Neighbor Embedding (tSNE) analysis on a panel including the phenotypic markers showed a unique color map axis gated on $\mathrm{T}$ cells in the CCs group compared with ATB and LTBI populations. By further gating on cells positive for each marker and then overlaying those events on top of the tSNE plots, their distribution suggested that some makers were differently expressed in the CCs group. Further analysis showed that the expression levels of CD69 on both $\mathrm{CD}^{+}$and $\mathrm{CD} 8^{+} \mathrm{T}$ cells were significantly lower in CCs group, especially on antigen-specific $\mathrm{T}$ cells. Thus, our data suggest that the activation status of CD69 might be associated with TB disease progression, and showed the potential to distinguish CCs from LTBI populations.

\section{MATERIALS AND METHODS}

\section{Subjects}

This study was approved by the Ethical Committee of Shanghai Pulmonary Hospital (approval number K18-215Z), and informed consent was obtained from all subjects. As shown in Table 1, the cohort comprised 45 ATB, 15 LTBI, 13 CCs and 17 non-TBinfected/close contacted persons (Non-TB). ATB was identified on the basis of sputum or effusion smear or polymerase chain reaction amplification positivity, confirmed by radiological findings and clinical syndromes, alongside with a final clinical diagnosis of ATB. LTBI was defined as IGRA-positive without clinical syndromes of active TB infection. CCs populations who were highly exposed to $M t b$ without infection fulfilled the following criteria: persons who had shared air space with an individual with pulmonary $\mathrm{TB}$ in the household or other indoor setting for $>15 \mathrm{hr}$ per week or $>180 \mathrm{hr}$ total during an infectious period (an infectious period was defined as the interval from 3 months before collection of the first culture-positive sputum specimen or the date of onset of cough, whichever was longer, through 2 weeks after the initiation of appropriate anti-tuberculosis treatment) and they were IGRA/TST negative without clinical syndromes of active TB infection. Non-TB subjects were defined as not $M t b$-infected (IGRA-negative) and not having had close contact with ATB patients. All subjects were $\mathrm{HIV} / \mathrm{HCV}$-negative and were not taking immunosuppressive drugs.

\section{Peripheral Blood Mononuclear Cells (PBMCs) Isolation}

An equal volume of heparinized peripheral venous blood and RPMI-1640 cell culture medium (Hyclone) were mixed and slowly added onto an equal volume of lymphocyte separation solution Ficoll at room temperature (HMK). The diluted whole blood was layered on top of the lymphocyte separation solution with a clear interface. After centrifugation at $600 \mathrm{x} g$ for $20 \mathrm{~min}$, PBMCs were collected and washed two times with RPMI 1640 medium. The cells were suspended in $1 \mathrm{~mL}$ R10 medium (RPMI1640 medium containing 10\% (v/v) fetal bovine serum [FBS]). Viability and numbers of cells were determined using trypan blue in a counting chamber.

\section{T-Spot Assay}

T-Spot assay uses an ELISpot (enzyme-linked immune absorbent spot) platform for diagnosing TB infection (Beijing Jinhao, China). The test was performed according to the instructions of the manufacturer (Li et al., 2017). Briefly, PBMCs were added into a 96-well plate which was pre-coated with antibodies against IFN- $\gamma$ in a volume of $100 \mu \mathrm{L} /$ well with or without ESAT6 and CFP10 peptides added as stimulants. After incubation at $37^{\circ}$ in a $5 \% \mathrm{CO}_{2}$ incubator for $18 \sim 20 \mathrm{~h}$, the wells were washed and a working solution of a second antibody conjugated to biotin was added (100 $\mathrm{LL} /$ well). After incubation for $1 \mathrm{~h}$ at room temperature, the wells were washed and the enzyme conjugate working solution was added (100 $\mu \mathrm{L} /$ well) and incubated for another $1 \mathrm{~h}$ at room temperature. After washing again, the chromogenic substrate AEC working solution was added $(100 \mu \mathrm{L} /$ well $)$ and incubated at room temperature for $7 \mathrm{~min}$ in the dark. Purified water was added to stop the reaction. The spots were counted by using an ImmunoSpot Reader (ChampSpot III, Beijing Sage Creation Science, China). 
TABLE 1 | Clinical characteristics of study participants.

\begin{tabular}{|c|c|c|c|c|}
\hline Groups & $\begin{array}{l}\text { Patients with Active } \\
\text { Tuberculosis }(n=45)\end{array}$ & $\begin{array}{l}\text { Latent Tuberculosis } \\
\text { Infection }(n=15)\end{array}$ & $\begin{array}{l}\text { Close Contacts } \\
\qquad(n=13)\end{array}$ & $\begin{array}{l}\text { Non-TB populations } \\
\qquad(n=17)\end{array}$ \\
\hline Age, yr & $41.82(14-77)$ & $43.27(17-73)$ & $44.02(33-75)$ & $43.71(23-76)$ \\
\hline \multicolumn{5}{|l|}{ Tuberculosis manifestation } \\
\hline Pulmonary & 33.33 & N/A & $\mathrm{N} / \mathrm{A}$ & N/A \\
\hline Extrapulmonary & 0 & $\mathrm{~N} / \mathrm{A}$ & $\mathrm{N} / \mathrm{A}$ & $\mathrm{N} / \mathrm{A}$ \\
\hline Both & 66.67 & $\mathrm{~N} / \mathrm{A}$ & $\mathrm{N} / \mathrm{A}$ & $\mathrm{N} / \mathrm{A}$ \\
\hline Smear/culture/ PCR positivity & 100 & 0 & $\mathrm{~N} / \mathrm{A}$ & $\mathrm{N} / \mathrm{A}$ \\
\hline \multicolumn{5}{|l|}{ Sex, \% } \\
\hline Male & 60 & 66.67 & 69.23 & 64.71 \\
\hline Female & 40 & 33.33 & 30.77 & 35.29 \\
\hline BCG vaccinated & 100 & 100 & 100 & 100 \\
\hline HIV & 0 & 0 & 0 & 0 \\
\hline
\end{tabular}

Data are median values (range) or percentage of participants. N/A, not applicable; PCR, polymerase chain reaction.

The results were defined as positive if the spots in the detection hole numbered at least 6 when the spots in negative control hole were fewer than 6 , or the spots in the detection well were twice as many as the spots in the negative control when the number in the negative control hole was greater than 6 .

\section{Intracellular Cytokine Staining (ICS) and Flow Cytometric Analysis}

The ICS assay was measured as described elsewhere (Hu et al., 2017). Briefly, the freshly isolated PBMCs were stimulated with peptide pools of ESAT- 6 and CFP10 $(5 \mu \mathrm{g} / \mathrm{mL}$, synthesized by GL Biochem, China) in the presence of Brefeldin A and Monensin (both $1 \mu \mathrm{g} / \mathrm{mL}, \mathrm{BD}$ Biosciences) in a roundbottom 96-well plate at $37^{\circ}$ and $5 \% \mathrm{CO}_{2}$ for $12 \mathrm{~h}$. After centrifugation at $600 \mathrm{xg}$ for $5 \mathrm{~min}$ at $4^{\circ}$, the cells were surfacestained with $50 \mu \mathrm{L}$ of the appropriate antibody panel diluted in PBS buffer containing $2 \%$ FBS for $30 \mathrm{~min}$ at $4^{\circ}$ in the dark, followed by washing, fixation and permeabilization with Cytofix/Cytoperm (BD Biosciences) for $20 \mathrm{~min}$ at $4^{\circ}$ in the dark. The fixed cells were washed then treated with a panel of antibodies against intracellular cytokines in a final volume of $50 \mu \mathrm{L}$ and incubated for another $30 \mathrm{~min}$ at $4^{\circ}$ in the dark. Finally, the cells were washed and transferred into the flow tube and analyzed by flow cytometry (LSR Fortessa, BD Biosciences). The data were analyzed by FlowJo 10 software (Ashland, OR).

\section{t-Distributed Stochastic Neighbor Embedding (tSNE) Analysis}

tSNE is a machine learning dimensionality-reducing algorithm that compares similarities of data points in high dimensional space and plots it in two dimensions through a way that preserves the structure of the dataset. In this study, firstly, all of the flow cytometry data from the ATB, LTBI and CCs groups were electronically concatenated into a single FCS file using FlowJo 10 software. Then the large concatenated files were down-sampled to create separate files for each group at a size of 50, 000 events/group. The equal-sized files were then gated on $\mathrm{CD}^{+} \mathrm{T}$ cells based on the antibodies of
CD3 and CD4, and tSNE analysis was performed in FlowJo using iterations $=1000$ and perplexity $=30$. The number of iterations refers to the number of loops in the repeated execution of the program, the default number $(=1000)$ was used in this study. Perplexity is associated with the number of nearest neighbors that are used in learning algorithms and can be regarded as a knob that sets the number of effective nearest neighbors in tSNE analysis. The most appropriate value depends on the density of the data, a relatively large value $(=30)$ was used since a large dataset was analyzed in this study.

\section{Antibodies}

The antibodies used in this study: CD3-PE/Dazzle594 (clone OKT3), CD38-FITC (clone 303504), CD69-Alexa Fluor 700 (clone 310922), Tim-3-Brilliant Violet 421 (clone F38-2E2) and CTLA-4-Percp-Cy5.5 (clone BN13) were from Biolegend. CD4PE-Cy5 (clone RPA-T4), HLA-DR-APC (clone LN3), PD-1-APCeFluor780 (clone EbioJ105) and IFN- $\gamma$-PE-Cy7 (clone 4S.B3) were from eBioscience.

\section{Statistical Analysis}

Statistical analyses were done using GraphPad Prism 7 software (La Jolla, CA). The data had a normal distribution and homogeneity of variance. The statistical differences between groups were assessed using one-way ANOVA tests. $P<0.05$ was considered statistically significant.

\section{RESULTS}

\section{Comparable T-Cell Frequencies Among ATB, LTBI, and CCs Groups}

To explore the role of $\mathrm{T}$ cells in close contacts of TB patients, we firstly compared the frequencies of $\mathrm{CD}^{+}$and $\mathrm{CD} 8^{+} \mathrm{T}$ cells among TB, LTBI and CCs groups (the gating strategy is shown in Supplementary Figure S1). As shown in Figures 1A,B, the percentages of $\mathrm{CD}^{+}$and $\mathrm{CD}^{+}{ }^{+} \mathrm{T}$ cells were comparable among groups as expected. 


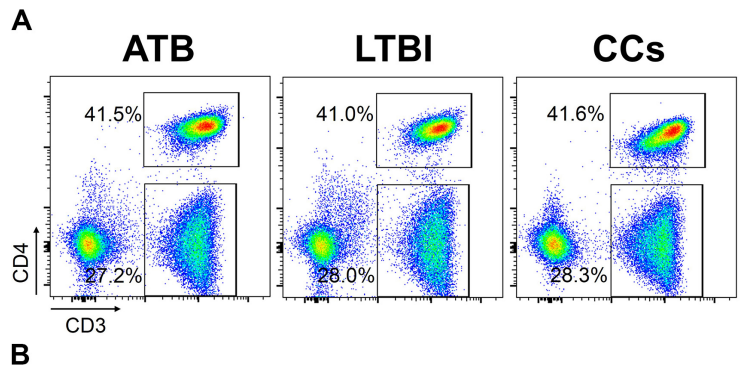

B
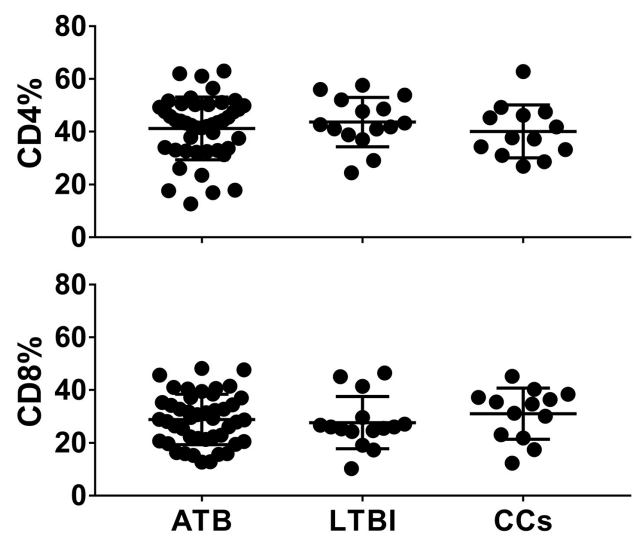

FIGURE 1 | The frequencies of $\mathrm{CD}^{+}{ }^{+}$and $\mathrm{CD} 8^{+}$T cell subsets among groups. The freshly isolated PBMCs were stimulated with peptide pools for $12 \mathrm{~h}$, and the percentage of CD4 ${ }^{+} \mathrm{T}$ cells and CD8 ${ }^{+} \mathrm{T}$ cells were determined by flow cytometry. Representative flow cytometric dot plots are shown in (A). $\mathrm{CD}^{+}{ }^{+} \mathrm{T}$ cells were gated as $\mathrm{CD} 3^{+} \mathrm{CD} 4^{+}$cells and $\mathrm{CD} 8^{+} \mathrm{T}$ cells were defined as $\mathrm{CD}^{+}{ }^{+} \mathrm{CD} 4^{-}$cells. Summarized data are shown as scatter dots in (B).

\section{tSNE Analysis of a Panel Including Several Phenotypic Markers on T Cells}

To further delineate the profiles of $\mathrm{T}$ cell-mediated immune responses in the CCs group, six T-cell biomarkers that had been reported to be associated with $M t b$ infection in mouse models were used. By gating on cells positive for each marker and then overlaying those events on top of the tSNE plots, we found that the cell distribution was similar between ATB and LTBI groups in a 2D scatterplot. Interestingly, the CCs group showed a unique plot map compared with the other two groups (Figure 2). This led us to further analysis of the different expression profiles of these markers.

\section{CD69 Is Under-Expressed on CD4 ${ }^{+}$and $\mathrm{CD}^{+}{ }^{+} \mathrm{T}$ Cells in CCs}

As shown in Figures 3A,B, the percentage of $\mathrm{CD}^{2} 9^{+}$cells among $\mathrm{CD}^{+} \mathrm{T}$ cells was significantly lower in household contacts and non-TB infected/close-contacted populations, compared with patients infected or latently infected with TB. The expression of CD69 showed no significant differences between the ATB and LTBI groups. The CCs and Non-TB groups also showed comparable CD69 expression on $\mathrm{CD}^{+}$ $\mathrm{T}$ cells (Figure 3). The differences between groups in mean fluorescence intensity (MFI) values of CD69 on $\mathrm{CD}^{+}{ }^{+} \mathrm{T}$ cells did not reach statistical significance (Supplementary Figure S2). Similarly, CD69 was also under-expressed on $\mathrm{CD}^{+} \mathrm{T}$ cells in CCs and Non-TB groups compared with TB infection groups (Figure 4). In contrast, the expression profiles of CD38, CTLA-4, HLA-DR, PD, and Tim-3 were comparable on both $\mathrm{CD}^{+} \mathrm{T}$ cells and $\mathrm{CD}^{+} \mathrm{T}$ cells, except that the expression of $\mathrm{CD} 38$ on $\mathrm{CD} 8^{+} \mathrm{T}$ cells was lower in CCs groups than in ATB patients (Figure 5). Thus, these data suggest that the activation status of CD69 is associated with Mtb infection and may have the potential to distinguish LTBI from those populations who are exposed continuously to $M t b$ but are not infected.

\section{The Expression of CD69 on IFN- $\gamma$-Responding T Cells}

To further characterize the association of CD69 with the status of TB infection, we tested the frequency of CD69 on Mtbspecific $\mathrm{T}$ cells, which were defined as $\mathrm{T}$ cells that responded to produce IFN- $\gamma$ after stimulation with $M t b$ antigens ESAT- 6 and CFP10. Although most CCs were IGRA-negative, they showed a slight IFN- $\gamma$ secretion at a background level in ICS assay, thus facilitating our analysis. Interestingly, our data showed that the expression levels of CD69 on IFN- $\gamma^{+} \mathrm{CD}^{+}{ }^{+} \mathrm{T}$ cells were significantly lower in the CCs group compared with the ATB $(P<0.01)$ and LTBI $(P<0.05)$ groups (Figure 6), which was a higher degree of significance than found with the total $\mathrm{CD} 4^{+}$ $\mathrm{T}$ cells (Figure 3). In addition, the $\mathrm{CD} 69^{+}$frequency was also significantly lower in IFN- $\gamma^{+} \mathrm{CD}^{+} \mathrm{T}$ cells in CCs compared with ATB $(P<0.05)$ and LTBI $(P<0.05)$ groups (Figure 7$)$.

Taken together, our data showed a lower expression of CD69 on T cells, especially on $M t b$ antigen-stimulated IFN$\gamma$-responding $\mathrm{T}$ cells, in the CCs group compared with TB infection groups, indicating that persistent CD69 activation is associated with the impaired host defense against $M t b$ infection that culminates in disease. It was recently shown that "resisters", defined as CCs with persistent TST/IGRA negativity, displayed enhanced antibody avidity and distinct $M t b$-specific IgG Fc profiles instead of IFN- $\gamma$ T-cell responses to ESAT-6 and CFP10 (Lu et al., 2019). Thus, our data support the contention that a CD69-expressing/IFN- $\gamma$-secreting non-T-cell host response to $M t b$ exposure might exist within the CCs population. Furthermore, lower CD69 expression in response to the $M t b$ antigen stimulation might be a clinically significant characteristic that could serve to distinguish CCs from TBinfected populations.

\section{DISCUSSION}

Despite significant progress over the past few decades, TB remains the world's leading killer infectious disease (WHO, 2019), suggesting that a more thorough understanding of the immune characteristics of $M t b$ infection is needed.

Especially needed are TB-specific biomarkers as indicators to predict infection reactivation, disease prognosis or vaccineinduced immune protection, and to differentiate various $\mathrm{TB}$ infection stages. Measurement of differences in T-cell activation 


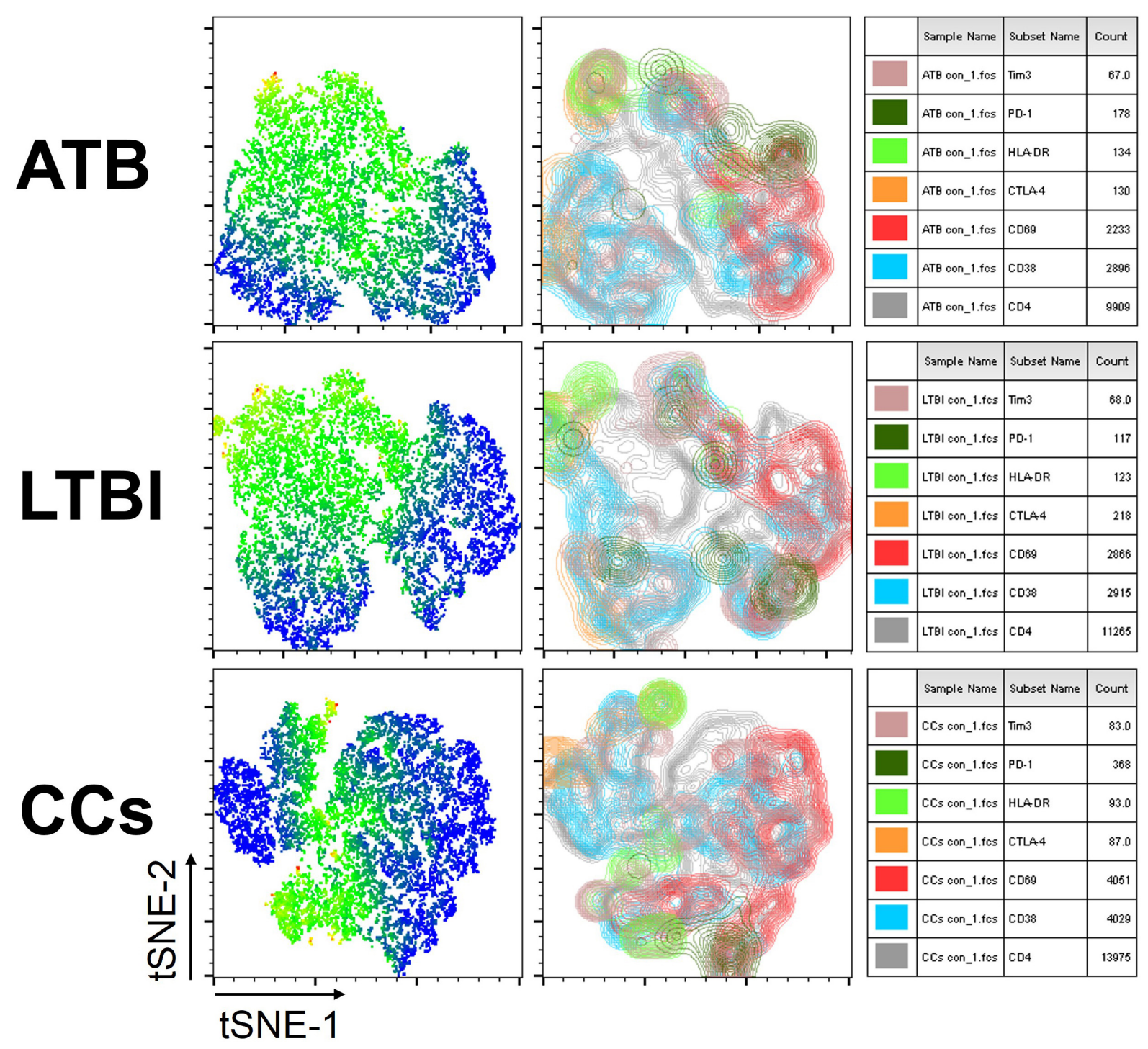

FIGURE 2 | tSNE map for six biomarkers on CD4+ T cells. The expression levels and distribution of different biomarkers in each group demonstrated by tSNE. The heat map shows the degree of activity of different cells expressing various biomarkers, the most active is indicated by red, and the least active is indicated by blue (left panel). The classic tSNE map indicates different signs with different colors, which more intuitively shows their expression on CD4 ${ }^{+} \mathrm{T}$ cells (middle panel). Combining heat map and classic map, the biomarkers for each color and the numbers of lymphocytes that express each biomarker are shown (right panel).

or exhaustion levels will be helpful. Previously, we demonstrated the role of $\mathrm{T}$-cell surface maker KLRG1 in human $\mathrm{CD}^{+}$ T-cell immunity against TB ( $\mathrm{Hu}$ et al., 2018). Recently, the concept of "resisters", defined as CCs who are persistently TST/IGRA negative, was described. "Resisters" were found to generate a non-IFN- $\gamma$-centric, $M t b$-specific, CD4 ${ }^{+}$T-cellmediated immune response to $\mathrm{TB}$ exposure, marked by high levels of up-regulated CD40L/CD154 in a clinical cohort study (Lu et al., 2019). This finding served to highlight the importance of T-cell surface marker expression in anti-TB T-cell-mediated immunity. Although a range of biomarkers was found associated with TB infection in mouse models, not all have been clinically verified and in particular, their expression profiles in CCs remain to be defined.
Our new finding, that CD69 was under-expressed in CCs group, suggested that it might be associated with $M t b$ infection. Considering that the sample size in our current cohort was not large, we also determined CD69's expression profiles in non$M t b$ infected/close contact (Non-TB) persons. Consistent with our expectation, the CD69's expression was significantly lower in the Non-TB persons compared with ATB and LTBI, further supporting our conclusion that activation status of CD69 on $\mathrm{T}$ cells was affected by $M t b$ infection.

CD69, a type II glycoprotein, is one of the earliest cell surface antigens expressed by $\mathrm{T}$ cells following activation, and potentially plays an important role in the activation and differentiation of a wide variety of hematopoietic cells (Ziegler et al., 1994). Numerous viral and bacterial infection models showed 

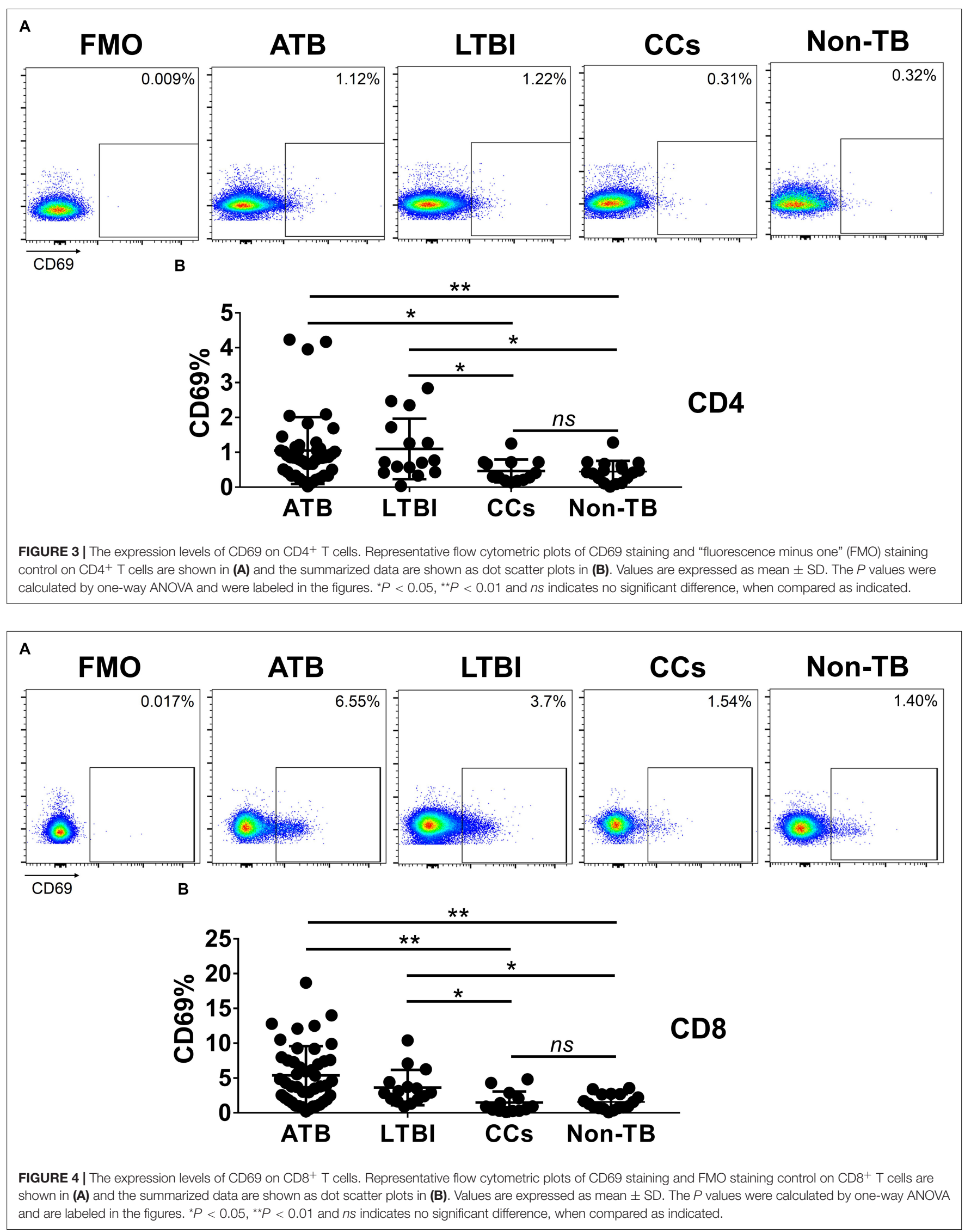


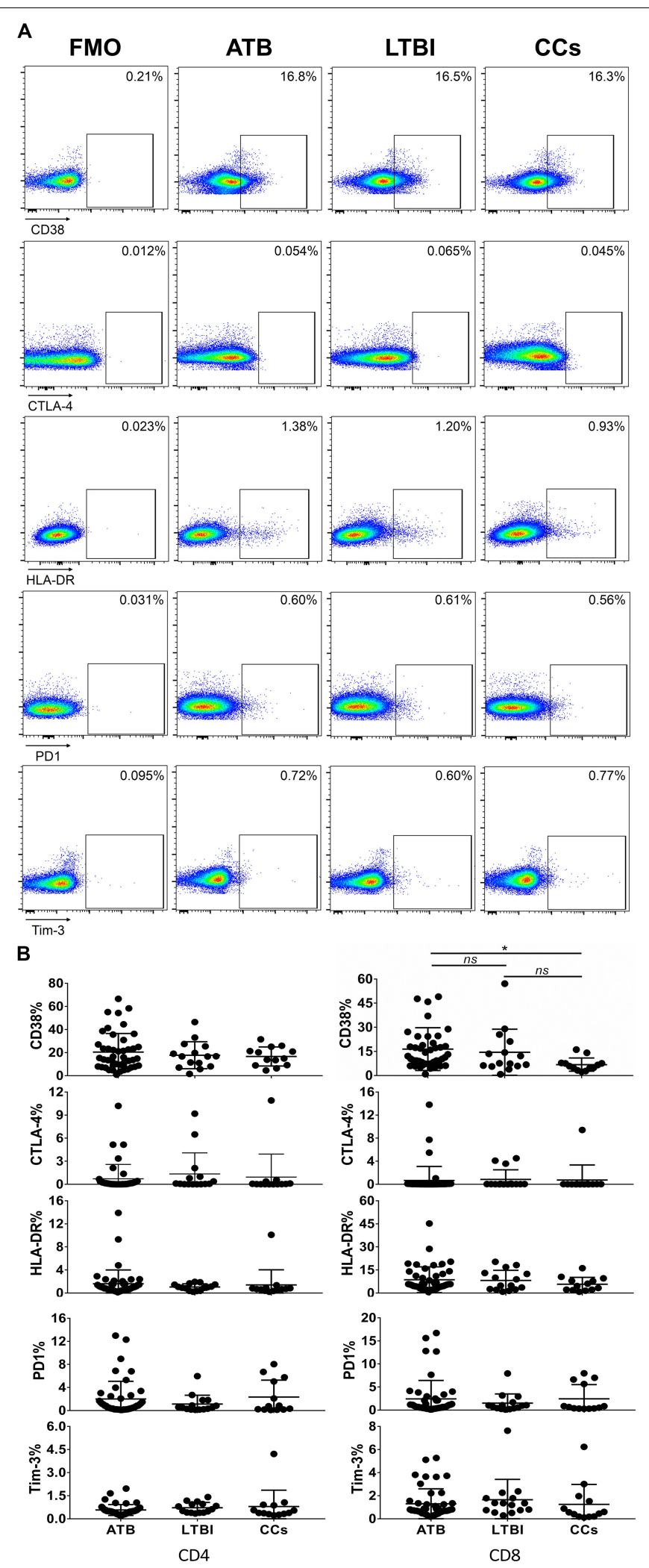

FIGURE 5 | The expression profiles of CD38, CTLA-4, HLA-DR, PD1, and Tim-3 on CD4 ${ }^{+}$T cells and CD8 ${ }^{+}$T cells. Representative flow cytometric plots of CD38, CTLA-4, HLA-DR, PD1, and Tim-3 staining and FMO staining controls are shown in (A) and the summarized data are shown as dot scatter plots in (B). Values are expressed as mean $\pm \mathrm{SD}$. The $P$ values were calculated by one-way ANOVA and are labeled in the figures. ${ }^{*} P<0.05$ and $n s$ indicates no significant difference, when compared as indicated. 


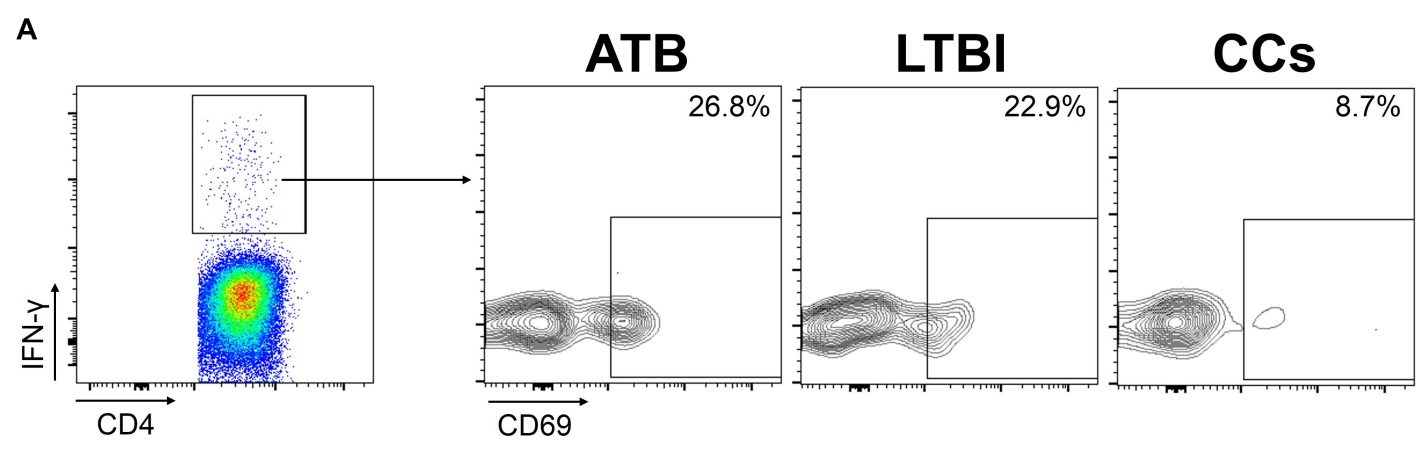

B
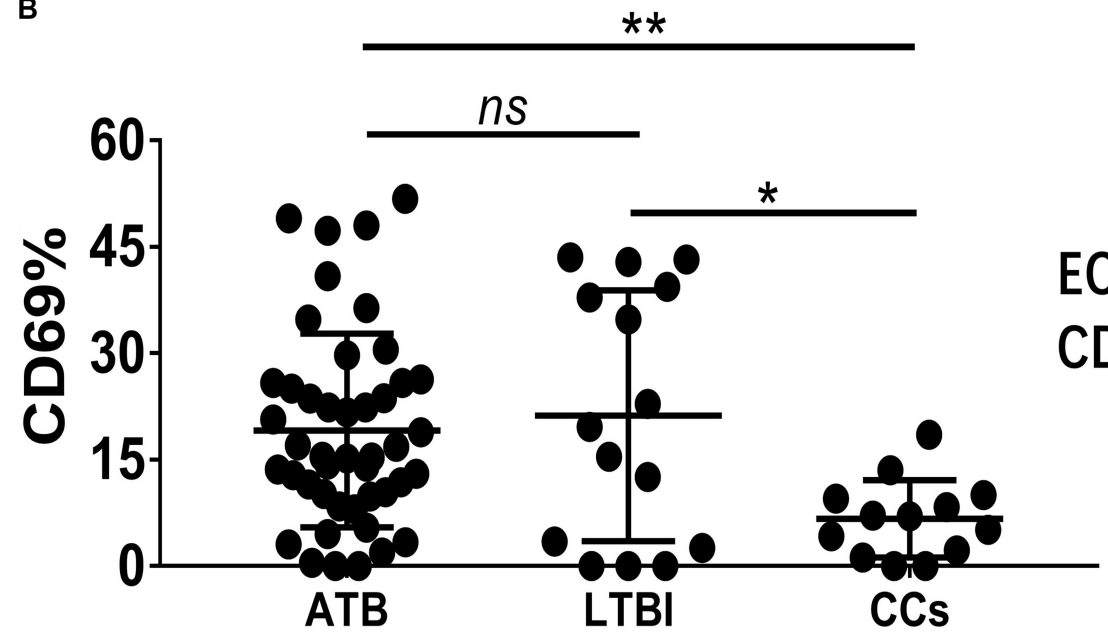

FIGURE 6 | The expression levels of CD69 on IFN- $\gamma$-responding CD4 ${ }^{+}$T cells. Representative flow cytometric plots of IFN- $\gamma$ and CD69 staining on CD4 ${ }^{+}$T cells are shown in (A) and the summarized data are shown as dot scatter plots in (B). EC, ESAT-6, and CFP10 peptide pools. Values are expressed as mean \pm SD. The $P$ values were calculated by one-way ANOVA and are labeled in the figures. ${ }^{*} P<0.05$, ${ }^{* *} P<0.01$ and $n s$ indicates no significant difference, when compared as indicated.

increased CD69 expression on T cells (Hodge et al., 2004; Vega-Ramos et al., 2010; Ishikawa et al., 2013). CD69 is also a co-stimulatory biomarker commonly expressed on $\mathrm{CD}^{+}$ $\mathrm{T}$ cells that bind to corresponding ligands that are expressed on DCs (Maimela et al., 2018). CD69 activation stimulates an influx of calcium ions and the activation of extracellular kinases ERK1/2, thereby facilitating $\mathrm{CD}^{+}{ }^{+} \mathrm{T}$ cell proliferation. In addition, CD69 activation stimulates the secretion of IL-2 and IFN- $\gamma$ that promotes the cytotoxic function of $\mathrm{CD}^{+} \mathrm{T}$ cells (Gonzalez-Amaro et al., 2013). CD69 is preferentially expressed on cells that activate the memory phenotype $\left(\mathrm{CD} 45 \mathrm{RO}^{+}\right.$ HLA-DR ${ }^{+}$), with an increase in the mucosal homing signal CCR6 and a decrease in the secondary lymphoid tissues homing signals CCR7 and CD62L. This suggests that CD69 may help lymphocytes migrate to the site of infection (Sallusto et al., 1999; Yong et al., 2017). The expression levels of CD69 on T cells fluctuated during anti-TB chemotherapy (Portales-Pérez et al., 2002) and in HIV-1/TB co-infected patients, the percentage of CD4+ T cells expressing CD69 ${ }^{+}$was related to TST and IGRA results (Hsieh et al., 2000) confirming an association with disease status. Because here we have demonstrated that there is increased CD69 expression on $\mathrm{CD}^{+}$and $\mathrm{CD}^{+} \mathrm{T}$ cell in ATB and LTBI we speculate that blockage of CD69 by a specific antibody might influence the function of $\mathrm{CD}^{+} \mathrm{T}$ cells. This remains to be investigated.

It was reported that primary viral infection might cause an IFN-I-dependent and systemic "partial" activation of $\mathrm{T}$ lymphocytes that was characterized by up-regulated expression of early activation marker CD69 and co-stimulatory molecule CD86 (Alsharifi et al., 2006). These partially activated T cells survived better and developed into effector cells more efficiently and thereby helped to eliminate virus infection in the early infection phase (Wijesundara et al., 2010). However, during chronic viral/bacterial infection and tumor formation, the ability of T cells to function and to establish memory might be impaired due to persistent/chronic antigen stimulation and then ultimately weaken the ability of the cells to confer host protection. This phenomenon was termed "T-cell exhaustion" (Blank et al., 2019). Exhausted $\mathrm{T}$ cells display high levels of surface markers such as PD-1, Tim-3, CTLA-4, LAG-3, and CD69 (Yi et al., 2010). Although the application of so-called "immune checkpoint blockade" therapies that target these molecules has become a major weapon in fighting cancer, a greater understanding of T-cell exhaustion is imperative to establish rational 

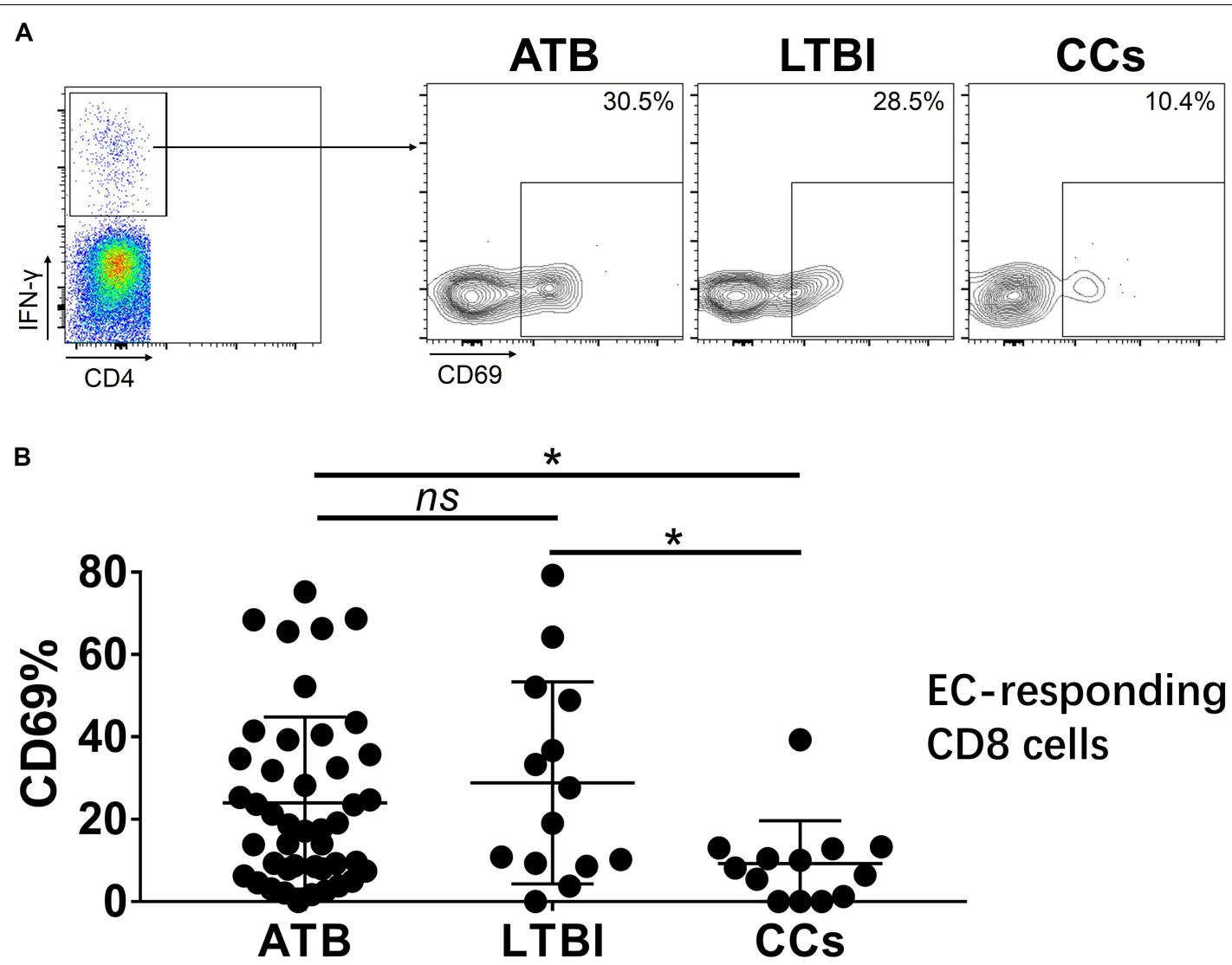

FIGURE 7 | The expression levels of CD69 on IFN- $\gamma$-responding $\mathrm{CD}^{+} \mathrm{T}$ cells. Representative flow cytometric plots of IFN- $\gamma$ and $\mathrm{CD} 69$ staining on $\mathrm{CD} 8^{+} \mathrm{T}$ cells are shown in (A) and the summarized data are shown as dot scatter plots in (B). Values are expressed as mean $\pm \mathrm{SD}$. The $P$ values were calculated by one-way ANOVA and were labeled in the figures. ${ }^{*} P<0.05$ and $n s$ indicates no significant difference, when compared as indicated.

immunotherapeutic interventions (Hashimoto et al., 2018). Previously, we showed that the expression of KLRG1, a maker of terminally differentiated $\mathrm{T}$ cells, was significantly increased during persistent $\mathrm{TB}$ infection, leading to an inadequate T-cell immune response ( $\mathrm{Hu}$ et al., 2018). With regard to CD69, it was reported that targeting CD69 enhanced the early control of virus infection which related to increased numbers of cytokine-producing $\mathrm{T}$ cells and $\mathrm{NK}$ cells in the periphery (Notario et al., 2019). The attenuation of tumor progression in CD69 knockout mice was related to the increased levels of tumor infiltrating $\mathrm{T}$ cells and the decreased levels of CD8 T-cell exhaustion, and anti-CD69 antibody treatment enhanced the anti-tumor activity (Mita et al., 2018). In this study, we showed that $M t b$ infection similarly led to increased levels of CD69 expression on T cells, whereas CCs population showed levels of CD69 expression similar to those in non-TB infected/close contacted population. Thus, our data suggest that persistent CD69 activation might impair host defense against $M t b$ infection.

Considering all the subjects were immunized with BCG vaccines, ESAT- 6 and CFP10, which are $M t b$-specific antigens and not secreted by BCG strains, were used throughout the study, to avoid potential confounding factors (Yang et al., 2013).
Even though, BCG-specific T-cell immune memory might also be associated with TB disease status, and it was showed that BCG increased CD69's expression on both CD4 (Hougardy et al., 2007) and natural killer cells (Marcenaro et al., 2008) in human cohort studies. Thus, it's reasonable to speculate that BCG-stimulated CD69's expression might also be used to define TB infection status, which worth further exploration.

It should be noted that CD8 $\mathrm{T}$ cells were roughly defined as $\mathrm{CD}^{+}{ }^{+} \mathrm{CD} 4{ }^{-}$cells in this study, however, several T-cell subsets such as $\gamma \delta \mathrm{T}$ cells, NKT cells and mucosal-associated invariant $\mathrm{T}$ cells might be $\mathrm{CD}^{+} \mathrm{CD}^{-} \mathrm{CD}^{-}$, and they are not rare in peripheral blood (D’Acquisto and Crompton, 2011). Indeed, these T-cell subsets have been shown to participate in human host defense against $M t b$ infection in patients with active TB (BehrPerst et al., 1999; Gansert et al., 2003; Jiang et al., 2014) and a decrease of CD69 expression on TCR Va7.2 ${ }^{+}$CD4 ${ }^{-}$T cells was reported to be associated with impaired cytotoxic functions in chronic hepatitis B virus-infected patients (Yong et al., 2017). Thus, the surface markers such as CD69 on these subsets also have the potential to differentiate between LTBI and CC groups, which is a possibility worth further evaluation.

In conclusion, this study demonstrated that an up-regulation of the T-cell phenotype that expresses CD69 might be associated 
with the progression of $M t b$ infection to disease. The potential of CD69 as an indicator to predict infection reactivation, to decrease immune protection, and to differentiate between CCs with LTBI and ATB remains to be further defined.

\section{DATA AVAILABILITY STATEMENT}

All datasets presented in this study are included in the article/Supplementary Material.

\section{ETHICS STATEMENT}

The studies involving human participants were reviewed and approved by the Ethical Committee of Shanghai Pulmonary Hospital (approval number K18-215Z), and informed consent was obtained from all subjects. The patients/participants provided their written informed consent to participate in this study.

\section{AUTHOR CONTRIBUTIONS}

$\mathrm{X}$-YF, WS, and $\mathrm{ZH}$ conceived and designed this study. LW, Z-YC, LG, and RQ collected clinical samples and performed

\section{REFERENCES}

Alsharifi, M., Regner, M., Blanden, R., Lobigs, M., Lee, E., Koskinen, A., et al. (2006). Exhaustion of type I interferon response following an acute viral infection. J. Immunol. 177, 3235-3241. doi: 10.4049/jimmunol.177.5.3235

Andersen, P., and Smedegaard, B. (2000). CD4(+) T-cell subsets that mediate immunological memory to Mycobacterium tuberculosis infection in mice. Infect. Immun. 68, 621-629. doi: 10.1128/iai.68.2.621-629.2000

Behr-Perst, S. I., Munk, M. E., Schaberg, T., Ulrichs, T., Schulz, R. J., and Kaufmann, S. H. (1999). Phenotypically activated gammadelta T lymphocytes in the peripheral blood of patients with tuberculosis. J. Infect. Dis. 180, 141-149. doi: $10.1086 / 314844$

Blank, C. U., Haining, W. N., Held, W., Hogan, P. G., Kallies, A., Lugli, E., et al. (2019). Defining 'T cell exhaustion'. Nat. Rev. Immunol. 19, 665-674.

D'Acquisto, F., and Crompton, T. (2011). CD3+CD4-CD8- (double negative) T cells: saviours or villains of the immune response? Biochem. Pharmacol. 82, 333-340. doi: 10.1016/j.bcp.2011.05.019

Dintwe, O. B., Day, C. L., Smit, E., Nemes, E., Gray, C., Tameris, M., et al. (2013). Heterologous vaccination against human tuberculosis modulates antigenspecific CD4+ T-cell function. Eur. J. Immunol. 43, 2409-2420. doi: 10.1002/ eji. 201343454

Gansert, J. L., Kiessler, V., Engele, M., Wittke, F., Rollinghoff, M., Krensky, A. M., et al. (2003). Human NKT cells express granulysin and exhibit antimycobacterial activity. J. Immunol. 170, 3154-3161. doi: 10.4049/ jimmunol.170.6.3154

Gonzalez-Amaro, R., Cortes, J. R., Sanchez-Madrid, F., and Martin, P. (2013). Is CD69 an effective brake to control inflammatory diseases? Trends Mol. Med. 19, 625-632. doi: 10.1016/j.molmed.2013.07.006

Hashimoto, M., Kamphorst, A. O., Im, S. J., Kissick, H. T., Pillai, R. N., Ramalingam, S. S., et al. (2018). CD8 t cell exhaustion in chronic infection and cancer: opportunities for interventions. Annu. Rev. Med. 69, 301-318. doi: 10.1146/annurev-med-012017-043208

Hodge, G., Hodge, S., Han, P., and Haslam, R. (2004). Multiple leucocyte activation markers to detect neonatal infection. Clin. Exp. Immunol. 135, 125-129. doi: $10.1111 /$ j.1365-2249.2004.02346.x experiments. ZH performed statistical analyses. X-YF, ZH, and $\mathrm{Z}$-YC interpreted the pooled results. $\mathrm{ZH}$ and Z-YC drafted the manuscript. X-YF and DL revised the manuscript. All authors approved the final manuscript.

\section{FUNDING}

This work was supported by Grants from the Chinese National Mega Science and Technology Program on Infectious Diseases (2018ZX10302301, 2018ZX10731301, and 2020ZX09201001006-002), National Natural and Science Foundation of China (81873884 and 31771004), and Shanghai Outstanding Academic Leaders Program (19XD1403100 and 2019LJ13).

\section{SUPPLEMENTARY MATERIAL}

The Supplementary Material for this article can be found online at: https://www.frontiersin.org/articles/10.3389/fmicb. 2020.01901/full\#supplementary-material

FIGURE S1 | Flow cytometry gating strategy. Representative flow cytometric dot plots are shown. Briefly, $\mathrm{CD} 4^{+} \mathrm{T}$ cells were gated as $\mathrm{CD} 3^{+} \mathrm{CD} 4^{+}$cells and $\mathrm{CD} 8^{+}$ T cells were defined as $\mathrm{CD}^{+} \mathrm{CD}^{-}$cells.

FIGURE S2 | The MFI values of CD69 on CD4 T cells are shown.

Hougardy, J. M., Verscheure, V., Locht, C., and Mascart, F. (2007). In vitro expansion of CD4+CD25highFOXP3+CD127low/- regulatory $\mathrm{T}$ cells from peripheral blood lymphocytes of healthy Mycobacterium tuberculosis-infected humans. Microbes Infect. 9, 1325-1332. doi: 10.1016/j.micinf.2007.06.004

Hsieh, S. M., Hung, C. C., Pan, S. C., Wang, J. T., Tsai, H. C., Chen, M. Y., et al. (2000). Restoration of cellular immunity against tuberculosis in patients coinfected with HIV-1 and tuberculosis with effective antiretroviral therapy: assessment by determination of CD69 expression on T cells after tuberculin stimulation. J. Acquir. Immune. Defic. Syndr. 25, 212-220. doi: 10.1097/ 00126334-200011010-00002

Hu, Z., Wong, K. W., Zhao, H. M., Wen, H. L., Ji, P., Ma, H., et al. (2017). Sendai virus mucosal vaccination establishes lung-resident memory CD8 T cell immunity and boosts BCG-primed protection against TB in mice. Mol. Ther. 25, 1222-1233. doi: 10.1016/j.ymthe.2017.02.018

Hu, Z., Zhao, H., Li, C., Liu, X., Barkan, D., Lowrie, D. B., et al. (2018). The role of KLRG1 in human CD4+ T-cell immunity against tuberculosis. J. Infect. Dis. 217, 1491-1503. doi: 10.1093/infdis/jiy046

Ishikawa, C., Kawakami, H., Uchihara, J., Senba, M., and Mori, N. (2013). CD69 overexpression by human T-cell leukemia virus type 1 Tax transactivation. Biochim. Biophys. Acta 1833, 1542-1552. doi: 10.1016/j.bbamcr.2013.03.006

Jayaraman, P., Jacques, M. K., Zhu, C., Steblenko, K. M., Stowell, B. L., Madi, A., et al. (2016). TIM3 mediates T cell exhaustion during Mycobacterium tuberculosis infection. PLoS Pathog. 12:e1005490. doi: 10.1371/journal.ppat. 1005490

Jiang, J., Wang, X., An, H., Yang, B., Cao, Z., Liu, Y., et al. (2014). Mucosalassociated invariant T-cell function is modulated by programmed death-1 signaling in patients with active tuberculosis. Am. J. Respir. Crit. Care Med. 190, 329-339.

Kaipilyawar, V., and Salgame, P. (2019). Infection resisters: targets of new research for uncovering natural protective immunity against Mycobacterium tuberculosis. F1000Res. 8.F1000.

Kauffman, K. D., Sallin, M. A., Hoft, S. G., Sakai, S., Moore, R., WilderKofie, T., et al. (2018). Limited pulmonary mucosal-associated invariant t cell accumulation and activation during Mycobacterium tuberculosis infection in rhesus macaques. Infect. Immun. 86:e0431-18. 
Kirman, J., McCoy, K., Hook, S., Prout, M., Delahunt, B., Orme, I., et al. (1999). CTLA- 4 blockade enhances the immune response induced by mycobacterial infection but does not lead to increased protection. Infect. Immun. 67, 37863792. doi: 10.1128/iai.67.8.3786-3792.1999

Lazar-Molnar, E., Chen, B., Sweeney, K. A., Wang, E. J., Liu, W., Lin, J., et al. (2010). Programmed death-1 (PD-1)-deficient mice are extraordinarily sensitive to tuberculosis. Proc. Natl. Acad. Sci. U.S.A. 107, 13402-13407. doi: 10.1073/pnas. 1007394107

Li, G., Li, F., Zhao, H. M., Wen, H. L., Li, H. C., Li, C. L., et al. (2017). Evaluation of a new IFN-gamma release assay for rapid diagnosis of active tuberculosis in a high-incidence setting. Front. Cell Infect. Microbiol. 7:117. doi: 10.3389/fcimb. 2017.00117

Lu, L. L., Smith, M. T., Yu, K. K. Q., Luedemann, C., Suscovich, T. J., Grace, P. S., et al. (2019). IFN- $\gamma$-independent immune markers of Mycobacterium tuberculosis exposure. Nat. Med. 25, 977-987. doi: 10.1038/s41591-019-0 441-3

Maimela, N. R., Liu, S., and Zhang, Y. (2018). Fates of CD8+ T cells in tumor microenvironment. Comput. Struct. Biotec. 17, 1-13. doi: 10.1016/j.csbj.2018. 11.004

Marcenaro, E., Ferranti, B., Falco, M., Moretta, L., and Moretta, A. (2008). Human NK cells directly recognize Mycobacterium bovis via TLR2 and acquire the ability to kill monocyte-derived DC. Int. Immunol. 20, 1155-1167.

Mita, Y., Kimura, M. Y., Hayashizaki, K., Koyama-Nasu, R., Ito, T., Motohashi, S., et al. (2018). Crucial role of CD69 in anti-tumor immunity through regulating the exhaustion of tumor-infiltrating T cells. Int. Immunol. 30, 559-567. doi: 10.1093/intimm/dxy050

Nandakumar, S., Kannanganat, S., Posey, J. E., Amara, R. R., and Sable, S. B. (2014). Attrition of T-cell functions and simultaneous upregulation of inhibitory markers correspond with the waning of BCG-induced protection against tuberculosis in mice. PLoS One 9:e113951. doi: 10.1371/journal.pone.011 3951

Notario, L., Redondo-Antón, J., Alari-Pahissa, E., Albentosa, A., Leiva, M., Lopez, D., et al. (2019). CD69 targeting enhances anti-vaccinia virus immunity. J. Virol. 93, e519-e553.

Phillips, B. L., Mehra, S., Ahsan, M. H., Selman, M., Khader, S. A., and Kaushal, D. (2015). LAG3 expression in active Mycobacterium tuberculosis infections. Am. J. Pathol. 185, 820-833. doi: 10.1016/j.ajpath.2014.11.003

Portales-Pérez, D. P., Baranda, L., Layseca, E., Fierro, N. A., de la Fuente, H., Rosenstein, Y., et al. (2002). Comparative and prospective study of different immune parameters in healthy subjects at risk for tuberculosis and in tuberculosis patients. Clin. Diagn. Lab. Immunol. 9, 299-307. doi: 10.1128/ cdli.9.2.299-307.2002

Reichler, M. R., Khan, A., Sterling, T. R., Zhao, H., Moran, J., McAuley, J., et al. (2018). Risk and timing of tuberculosis among close contacts of persons with infectious tuberculosis. J. infect. Dis. 218, 1000-1008.

Reiley, W. W., Shafiani, S., Wittmer, S. T., Tucker-Heard, G., Moon, J. J., Jenkins, M. K., et al. (2010). Distinct functions of antigen-specific CD4 T cells during murine Mycobacterium tuberculosis infection. Proc. Natl. Acad. Sci. U.S.A. 107, 19408-19413. doi: 10.1073/pnas.1006298107
Sallusto, F., Lenig, D., Forster, R., Lipp, M., and Lanzavecchia, A. (1999). Two subsets of memory T lymphocytes with distinct homing potentials and effector functions. Nature 401, 708-712. doi: 10.1038/44385

Samanovic, M. I., and Darwin, K. H. (2016). Game of 'Somes: protein destruction for Mycobacterium tuberculosis pathogenesis. Trends Microbiol. 24, 26-34. doi: 10.1016/j.tim.2015.10.001

Simmons, J. D., Stein, C. M., Seshadri, C., Campo, M., Alter, G., Fortune, S., et al. (2018). Immunological mechanisms of human resistance to persistent Mycobacterium tuberculosis infection. Nat. Rev. Immunol. 18, 575-589. doi: 10.1038/s41577-018-0025-3

Vega-Ramos, J., Alari-Pahissa, E., Valle, J. D., Carrasco-Marín, E., Esplugues, E., Borràs, M., et al. (2010). CD69 limits early inflammatory diseases associated with immune response to Listeria monocytogenes infection. Immunol. Cell Biol. 88, 707-715. doi: $10.1038 /$ icb. 2010.62

WHO (2019). Global Tuberculosis Report 2019. Geneva: WHO.

Wijesundara, D. K., Kumar, S., Alsharifi, M., Mullbacher, A., and Regner, M. (2010). Antigen-specific activation thresholds of CD8+ T cells are independent of IFN-I-mediated partial lymphocyte activation. Int. Immunol. 22, 757-767. doi: 10.1093/intimm/dxq064

Workman, C. J., Cauley, L. S., Kim, I. J., Blackman, M. A., Woodland, D. L., and Vignali, D. A. (2004). Lymphocyte activation gene-3 (CD223) regulates the size of the expanding $\mathrm{T}$ cell population following antigen activation in vivo. J. Immunol. 172, 5450-5455. doi: 10.4049/jimmunol.172.9.5450

Yang, J., Xu, K., Zheng, J., Wei, L., Fan, J., and Li, L. (2013). Limited T cell receptor beta variable repertoire responses to ESAT- 6 and CFP-10 in subjects infected with Mycobacterium tuberculosis. Tuberculosis 93, 529-537. doi: 10.1016/j.tube. 2013.05.007

Yi, J. S., Cox, M. A., and Zajac, A. J. (2010). T-cell exhaustion: characteristics, causes and conversion. Immunology 129, 474-481. doi: 10.1111/j.1365-2567. 2010.03255.X

Yong, Y. K., Tan, H. Y., Saeidi, A., Rosmawati, M., Atiya, N., Ansari, A. W., et al. (2017). Decrease of CD69 levels on TCR Valpha7.2(+)CD4(+) innatelike lymphocytes is associated with impaired cytotoxic functions in chronic hepatitis B virus-infected patients. Innate. Immun. 23, 459-467. doi: 10.1177/ 1753425917714854

Ziegler, S. F., Ramsdell, F., and Alderson, M. R. (1994). The activation antigen CD69. Stem Cells 12, 456-465. doi: 10.1002/stem.5530120502

Conflict of Interest: The authors declare that the research was conducted in the absence of any commercial or financial relationships that could be construed as a potential conflict of interest.

Copyright (c) 2020 Chen, Wang, Gu, Qu, Lowrie, Hu, Sha and Fan. This is an open-access article distributed under the terms of the Creative Commons Attribution License (CC BY). The use, distribution or reproduction in other forums is permitted, provided the original author(s) and the copyright owner(s) are credited and that the original publication in this journal is cited, in accordance with accepted academic practice. No use, distribution or reproduction is permitted which does not comply with these terms. 